\title{
Society of Cardiovascular Anesthesiologists 31st annual meeting highlights
}

\author{
Colleen Koch, MD
}

San Antonio, Texas, was the setting for the Society of Cardiovascular Anesthesiologists (SCA) 31st annual meeting. The program committee organized a scientific program with high-caliber national and international faculty that broached diverse topics pertinent to both clinicians and basic scientists. The interdisciplinary nature of the program was reflected by invited faculty from surgery, basic science, cardiology, critical care medicine, and perfusion. The topics of refresher courses included challenges of geriatric cardiac surgery, advances in cardiac imaging, considerations in blood management, and issues in cerebral monitoring.

Basic science sessions provided insight into work in the experimental bypass laboratory with such topics as "Downsizing: What Have We Learned from Rodent Models of Cardiopulmonary Bypass?' Subspecialty societies included the Society of Critical Care Anesthesiologists, which offered sessions on sedation and resuscitation guidelines; Society for the Advancement of Geriatric Anesthesia presented " 85 Is the New 65," covering topics pertinent to understanding autonomic and vascular dysfunction in the elderly; the Pediatric Cardiac Anesthesia group provided updated sessions on management issues with congenital heart disease. In the area of heart failure, updates on continuous flow mechanical circulatory devices and total artificial hearts were provided. Risk scores for ventricular assist device implantation as well as the usefulness of left ventricular assist device as destination therapy were discussed. A number of diverse educational offerings included sessions on ethics and professional development with SCA Data on Physician Reimbursement, clinical controversies, genomics, and future treatment implications and preconditioning in clinical practice. Practical sessions included pro/con debates on patent foramen ovale closure in off-pump coronary artery bypass grafting, use of antifibrinolytics in clinical practice, and clinical applications for use of recombinant factor 7 .

The annual meeting included interactive workshops on intermediate and advanced transesophageal echocardiography (TEE) that included applications of tissue Doppler, strain, and 3-D echocardiography as well as practical applications for practicing cardiothoracic anesthesiologists. There were additional well-attended interactive workshops for handheld ultrasound for venous access, thoracic anesthesia, essentials of cardiopulmonary bypass, and point-of-care testing. A highlight of the program was the session on FOCUS update with guest lecturer Peter J. Pronovost, MD. Further information was provided by Dr Speiss in "The Next Steps to Continue Our Focus on Human Error." Future trends in anesthesia and acute kidney injury and updates on mechanical adjuncts and pharmacologic therapies to promote cerebral protection for patients having deep hypothermic circulatory arrest were also featured in the sessions.

The program committee was honored to have keynote speaker Dr Larry Cohn deliver a lecture on "Mitral Valve Repair: The Perfect Collaboration of Cardiologist, Cardiovascular Anesthesiologist and Cardiac Surgery." Dr. Cohn's years of experience and insight were reflected in his well-received lecture emphasizing the importance of collaboration for optimal patient management.

Of note, a first-time Introduction to TEE (iTEE) course was offered concurrent with the annual meeting workshops and refresher course lectures. iTEE was specially designed for anesthesiologists seeking fundamental principles and applications of TEE for the non-cardiac surgery practice. The meeting concluded with the morning devoted to interactive sessions on 3-D TEE and point-of-care testing.

From the Cleveland Clinic Foundation, Cleveland, Ohio.

Address for reprints: Colleen Koch, MD, The Cleveland Clinic Foundation, Cardiothoracic Anesthesia (G-3), 9500 Euclid Avenue, Cleveland, OH44195 (E-mail: kochc@ccf.org).

J Thorac Cardiovasc Surg 2009;138:798

$0022-5223 / \$ 36.00$

Copyright (c) 2009 by The American Association for Thoracic Surgery

doi:10.1016/j.jtcvs.2009.06.009 